\title{
Monitoring and Evaluation of Fetal Heart Rate via iPhone
}

\author{
Gábor Sipka, Tibor Szabó, Ráhel Zöllei-Szénási, Melinda Vanya, Mária Jakó, \\ Tamás Dániel Nagy, János Borbás, Márta Fidrich, Tamás Bitó, György Bártfai \\ Department of Obstetrics and Gynecology, University of Szeged, \\ Semmelweis str. 1, H-6725 Steged, Hungary \\ jmarica8@gmail.com
}

\begin{abstract}
The recording of fetal heart rate can be reassuring for the mother about the fetus' wellbeing. Our smart phone application can detect, record and evaluate fetal heart rate at any time. This method is radiation-free, and can be used all day without harming the fetus. It does not require medical assistance and easy to use at home. It reduces the queue at outpatient care units, helps pregnant women to relieve stress by listening to their unborn baby's heartbeat. It improves mother- child relationship yet sends an alarming message if further examinations are needed to prevent the consequences of hypoxia.
\end{abstract}

Keywords: fetal heart rate, mobile application, home monitoring, phonocardiograohy

\section{Introduction}

There are several solutions for the acustic detection of fetal heart rate that can help obstetricians determine whether the fetus is at risk or not [1, 3-5]. The latest protocol suggests two occasions to examine the fetal heart. The first is performed by ultrasound on the $18-21^{\text {st }}$ week of pregnancy and the purpose is to investigate the anatomy of the heart and main vessels. From the $38^{\text {th }}$ week (or incase of any complications from the $24^{\text {th }}$ week) cardiotocograph (CTG) is used to monitor the changes and characteristics of fetal pulse and movements. Two transducers are placed on the maternal abdomen; one to detect fetal heart actions (cardio-) the other registers the uterine activity (tocogram). This way the heart rate is detected by Doppler ultrasound while the uterine contractions via a pressure-sensitive contraction transducer, called tocodynamometer. The pressure required to flatten a section of the wall correlates with the internal pressure, thereby providing an estimate to it. Phonocardiography (PCG) is also proper way to detect fetal heartbeat. The device can record the rhythmic contractions due to the resonance made by the valves. A healthy fetal heart rate is between 120 and 160 beats per minute, a low (bradycardia) or high (tachycardia) heart rate indicates the necessity of other examinations or termination of pregnancy. Counting of fetal movements gains importance from the $24^{\text {th }}$ week and it also reliable indicator of fetal wellbeing.

DOI: http://dx.doi.org/10.1007/978-3-319-49655-9 60

Published version 
The variability of the fetal heart rate provides valuable information about central nervous system. This component can be different on the 20-30 minutes long registration period depending on the fetal daily behavioral rhythm. There are four main behavioral patterns that can alternate during each day. $1 \mathrm{~F}$ is defined as deep sleep with no major movements, $2 \mathrm{~F}$ is called REM sleep characterized by eye movements but no or rare body movements. The awake fetus can rest calmly moving only extremities or head $(3 \mathrm{~F})$ or can playfully explore the womb, moving with whole body, kicking and boxing around (4F). These different activities naturally affect the fetal pulse and oxygen need.

Brown and Patrick (1981) showed that apart from fetal activity and normal basic heart rate the alteration of higher and lower heart rates is also important factor to evaluate the fetal state [2]. They have set up some alternatives for ultrasonographic non-invasive fetal heart rate monitoring such as fetal electrocardiography (fECG), fetal magnetocardiography (fMCG), fetal phonocardiography (fPCG). The fECG requires several electrodes and the form of the recorded waves highly depends on their positions. The fMCG is too big and expensive for long time homemonitoring. The fPCG has unquestionable advantages for a safe long lasting use at home environment.

\section{Materials and Methods}

There are numerous electric stethoscopes available (Welch-Allyn, 3M Littmann, Thinklabs, Cardionics) but the prices go from 200 to 600 EUROs [4]. Our aim was to develop a small, cheap, non-invasive device that can easily be used at home to record fetal heart rate constantly.

Our smart phone application can record evaluate and replay fetal heart rate. A modified stethoscope head drives the signal (filtered from the mother's heart beat, breathing or bowel sound) to the telephone. The software then records it and shows the number of beats per minute real time, variability and plays an alarming sound in case of unsatisfying signal. Only the spectral separation method resulted in clear recordings, the acceleration sensor or the piezo sensor was not successful for this method $[1,3,5]$. After the signal proceeding an algorithm was developed to evaluate the heartbeat. Figure 1 shows screenshots of the running application.

DOI: http://dx.doi.org/10.1007/978-3-319-49655-9 60

$\underline{\text { Published version }}$ 

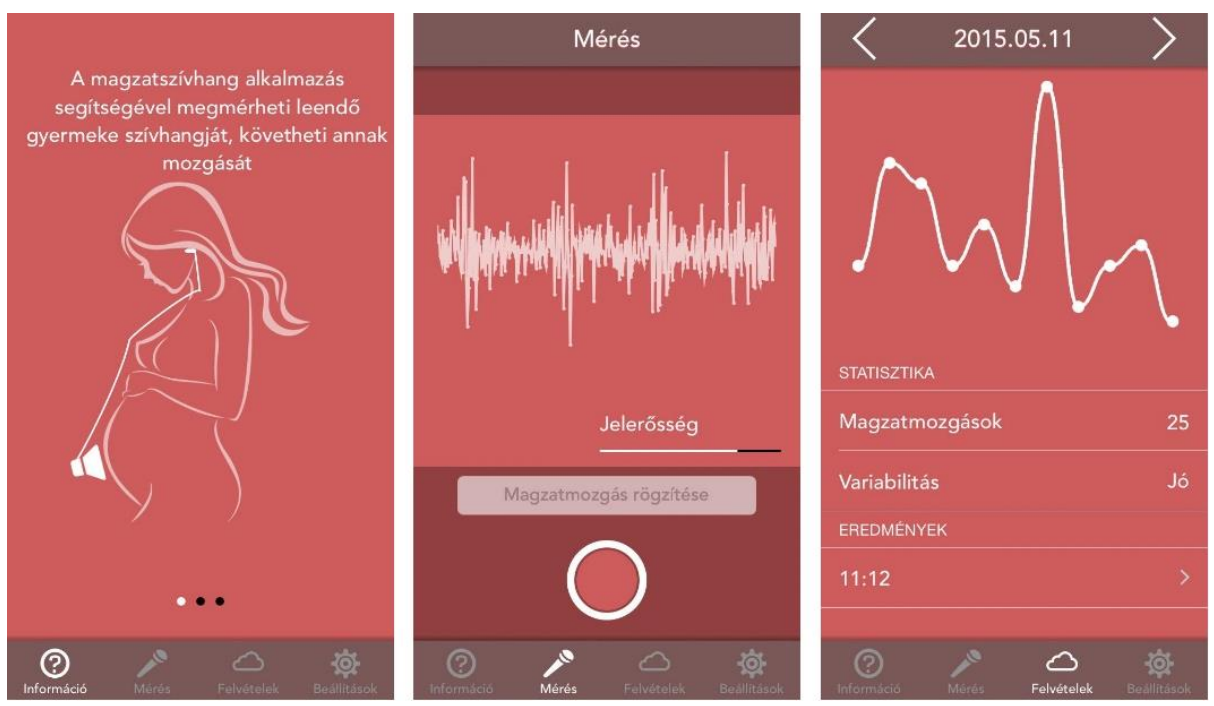

Fig. 1. With this application you may listen to your future child's heartbeat and movements and you may have an evaluation of it to make sure that your child is healthy. Menu: Informatin, Record, Recordings, Settings. The second screenshot shows the application in work; the recording, the signal, the fetal movement button, and the menu. The third screenshot is the statistic evaluation of fetal movements; date, recording, fetal movements (25), evaluation (good), start of the recording, menu.

\section{Discussion}

This phonocardiographic recording does not require healthcare assistance and still can provide signals of excellent quality. Although the usability of the heart rate recording depends on the relative position of the stethoscope head to the fetal heart, it is sensitive enough to let you listen to your baby's heart at any time all day. The method is completely passive, it does not radiates or harms your fetus. You may use it permanently or for a shorter time at a family event. Helps you make sure your fetus is healthy, prevents dysfunctions caused by oxygen deficit. You may send the saved recordings to your doctor who can immediately provide you a diagnosis. You will not need to queue in front of the outpatient care unit. It helps you relieve stress and it is very heartwarming listening to you baby. It improves mother-baby relationship and helps the whole family to prepare to welcome the new member.

Acknowledgments. This study was supported by "Telemdicine focused Researches on the Fields of Medicine, Mathematics and Informatics" TÁMOP- 4.2.2.A11/1/KONV-2012-0073 project. The research was financed by the European Union and the European Social Fund. 


\section{References}

1. Zukerwar, A., Pretlow, R., Stoughton, J., Baker, D.: Development of a piezo-polymer pressure sensor for a portable fetal heart rate monitor, IEEE Trans. Biomed. Eng. 40, 9, $963-$ 969 (1993)

2. Brown, R., Patrick, J.: The nonstress test: How long is enough?, Am J Ob Gyn. 151, 646$651(1981)$

3. Talbert, D.G., Davies, W.L., Johnson, F., Abraham, N., Colley, N., Southall, D.P.: Wide band with fetal phonography using a sensor matched to the compliance of the mother's abdominal wall, IEEE Trans. Biomed. Eng. 33, 175-181 (1986)

4. Andrés, E., Hajjam, A., Brandt, C.,: Advances and innovations in the field of auscultation, with a special focus on the development of new intelligent communicating stethoscope systems, Health Technol. 2, 5-16 (2012)

5. Goovaerts, H.G., Rompelman, O., van Geijn, H.P.: A transducer for detection of fetal breathing movements, IEEE Trans. Biomed. Eng. 36, 471-478 (1989)

6. Nagel, J.: New diagnostic and technical aspects of fetal phonocardiography, Eu J Ob Gyn Rep Biol, 23, 295-303 (1986)

DOI: http://dx.doi.org/10.1007/978-3-319-49655-9 60

Published version 